\title{
Effet de la salinité sur la croissance des deux variétés de bananier " grande naine » et " petite naine » et leur nutrition minérale au Maroc
}

\author{
Meriem BELFAKIH ${ }^{1 *}$, Mohammed IBRIZ1', Abdelmjid ZOUAHRI2 et Said HILALI ${ }^{3}$ \\ ${ }^{1}$ Laboratoire de Génétiques Biométrie, Faculté des sciences, Université Ibn Tofail, BP 133. 14 000. Kenitra. \\ 2 Unité de Recherche "Environnement et Conservation des Ressources Naturelles », INRA, CRRA de Rabat, BP \\ 6356 Rabat Instituts, Maroc \\ 3 Unité de recherche en Agro-Ressources Marocaines et Environnement Université Hassan Premier Faculté des \\ Sciences et Techniques Settat Maroc.
}

"Correspondance, courriel : m_belfakih2000@yahoo.fr

Original submitted in on 19th December 2012. Published online at www.m.elewa.org on 30th March 2013.

\section{RÉSUMÉ}

Objectifs : Le travail présenté ici a pour objectif de décrire l'effet de la salinité sur la croissance et la distribution des principaux éléments minéraux chez le bananier.

Méthodologie et résultats : L'étude de l'effet de la salinité a été réalisée sur deux variétés du bananier (Musa acuminata $L$ ), la grande naine et la petite naine issues de culture in vitro. Les variétés testées ont été combinées à quatre niveaux de chlorure de sodium $(\mathrm{NaCl})$ à savoir : $0,2,4$ et 6 grammes par litre $(\mathrm{g} / \mathrm{l})$. Les résultats ont montré la réduction de la biomasse aérienne et racinaire, une accumulation de potassium dans la partie aérienne et de sodium dans la partie racinaire, ainsi qu'une réduction d'absorption de calcium et de magnésium et une légère variation d'azote dès la concentration $4 \mathrm{~g} / \mathrm{l}$. Une certaine tolérance aux sels a été notée chez la variété grande naine comparativement à la petite naine. Cette tolérance pourrait être liée à une bonne sélectivité vis-à vis du potassium. Les variétés étudiées se comportent comme des plantes exclusives.

Conclusion et application de la recherche: Les caractéristiques ioniques de la variété de bananier, la grande naine, diffèrent de celles de la petite naine, Les résultats obtenus confirment que l'amélioration de la productivité des bananiers au Maroc ne peut être réalisée que par des travaux destinés à élaborer un matériel génétique adapté à partir de la variété grande naine. Le choix doit s'effectuer sur la base d'un grand nombre de caractères (morphologiques, biochimiques physiologiques) contrôlant les déterminismes génétiques complexes de la tolérance à la salinité.

Mots clés : Bananier, chlorure de sodium, éléments minéraux. 


\begin{abstract}
Effect of salinity on the growth of two varieties of banana Grande Naine" and "Petite Naine " and their mineral nutrition in Morocco

Objectives: The present work is intended to describe the effect of salinity on the growth and distribution of major minerals in banana.

Methods and results: The study of the effect of salinity was carried out on two banana varieties (Musa acuminata $L$ ), the grand Naine and the petite Naine from in vitro culture. The varieties tested were combined with four levels of $\mathrm{NaCl}(0,2,4$ and 6 gal / I). Results showed the reduction of biomass and root accumulation of potassium in the shoot and sodium in the root. There was reduced absorption of calcium and magnesium and a slight variation from the nitrogen concentration of $4 \mathrm{~g} / \mathrm{l}$. Some salt tolerance was noted in the Grande Naine compared to the petite Naine variety. This tolerance may be associated with a good selectivity towards potassium.

Conclusion and application of research: ionic characteristics of the banana variety, the Grande Naine differs from the petite Naine. The results confirm that improvements in the productivity of banana in Morocco should be achieved by work to develop Grande Naine germplasm by the use of a large number of characters (morphological, biochemical, physiological) controlling complex genetic determinism of salinity tolerance
\end{abstract}

Keywords: Banana, sodium chloride, minerals.

\section{INTRODUCTION}

Le bananier (Musa sp.) fruit introduit au Maroc au début des années 1940 en tant que plante ornementale. Sa culture n'a commences à retenir l'attention des producteurs qu'après l'arrêt de l'importation de la banane en 1977 (SRPV/MARA 1981). Le bananier, espèce aux exigences tropicales, est un arbre fruitier dont la production occupe la troisième place mondiale, après celle des agrumes et de la vigne (DPV/DH/MARA 1993). La culture du bananier a connu un développement important au Maroc (Morchid, 1999), elle est pratiquée principalement dans le nord-ouest (le Gharb), l'ouest (sud de Casablanca) et le sud (Souss-Massa) (CA/MADR 2003). La culture s'est concentrée surtout dans cette dernière zone, avec 1500 ha en 1997, suivie de la zone du Gharb (650 ha) (Morchid, 1999). La production en plein champ est limitée à la seule région du Sous Massa et en particulier, dans les plantations traditionnelles de Tamri et de Tamraght (DPV/DH/MARA 1993).Selon Galàn et al. , (2004), le Maroc est considéré comme le premier producteur mondial de bananes sous serre avec 4.460 ha, suivi de l'Espagne avec 3.000 ha. Ainsi,
5.683 ha ont été récoltés en 2008 avec une production de 214.712 tonnes (www.faostat.fao.org ). Les variétés de bananes à dessert utilisées sont de type "Musa Cavendish». Les variétés les plus cultivées au Maroc sont la " Grande Naine », avec $82 \%$, suivie de la « Petite Naine », avec $10 \%$, et la "Williams" avec seulement $7 \%$ de la superficie totale (Anonyme 2005). Cependant la culture du bananier est exposée à de nombreux problèmes entre autres la salinité excessive des sols et des eaux d'irrigation qui entraine surtout des faibles pourcentages de levée, ainsi que des baisses de rendements. Dans plusieurs régions du monde et plus particulièrement dans les zones arides et semiarides, la salinité prend de plus d'ampleur. La superficie affectée par le sel à l'échelle mondiale est de l'ordre de 955 millions (Szablocs, 1994). Au Maroc dans les périmètres irrigués, la salinité des sols et des eaux d'irrigation affecte environ $38 \%$ de la superficie totale (Ftouhi, 1981). La salinité du sol réduit la croissance et la productivité de la culture en raison de la diminution de la pression osmotique dans le sol et de l'augmentation de la 


\section{Belfakih et al... J. Appl. Biosci. 2013. Effet de la salinité sur la croissance des bananier et leur nutrition minérale au Maroc}

concentration de certains ions, qui atteint alors un niveau toxique pour la plante. Ces facteurs interfèrent non seulement avec des processus physiologiques tels que la transpiration, la photosynthèse, la translocation et la respiration, mais de plus, ils provoquent un déséquilibre hydrique et/ou ionique chez la plante (Richards 1992, Bohra et al. 1993). La survie et la croissance des végétaux sont liées au transport et la compartimentation des ions, à la biosynthèse et l'accumulation d'osmolyte organiques qui participent à l'ajustement osmotique et aux remaniements protéiques nécessaires à l'intégrité cellulaire (Greenway et Munns, 1980). Parmi les plantes adaptées au stress salin, on distingue les plantes exclusives, qui n'accumulent pas l'ion $\mathrm{Na}+$ dans les parties aériennes et de l'utiliser pour régler le potentiel osmotique cellulaire (Storey et

\section{MATÉRIELS ET MÉTHODES}

L'étude de l'effet de la salinité a été réalisée sur deux variétés du bananier (Musa acuminata $\mathrm{L}$ ), la grande et la petite naine issues de culture in vitro. Ces vitroplants ont été ramenés d'une serre de culture du bananier située dans la région du Gharb. Le dispositif expérimental adopté est de type factoriel à deux facteurs (variété, salinité) et trois répétitions. Les vitro plants issus de laboratoire sont plantés dans des sacs de polyéthylène noir de $20 \mathrm{~cm}$ de long et $12 \mathrm{~cm}$ de diamètre contenant de la tourbe. Les pots sont maintenus au voisinage de l'humidité à la capacité au champ $(\mathrm{Hcc})$ par des arrosages réguliers à raison d'un jour sur deux. Les variétés testées ont été combinées à quatre niveaux de $\mathrm{NaCl}(0,2,4$ et $6 \mathrm{gl} / \mathrm{l})$. Les teneurs en azote $(\mathrm{N})$, phosphore $(\mathrm{P})$, potassium $(\mathrm{K}+)$, calcium $(\mathrm{Ca}++)$, sodium $(\mathrm{Na}+)$, magnésium $(\mathrm{Mg}++)$ ont été déterminées dans le laboratoire de chimie du sol, a I'Institut National de Recherche Agronomique Rabat.

Dosage des éléments minéraux : Dosage du sodium et potassium (Benmiloud et Poloczanka, 1977)

Méthode et principe : Le $\mathrm{K}+$ et $\mathrm{Na}+$ sont déterminés au moyen d'un photomètre à flamme de marque JENWAPER7.

Les teneurs en $\mathrm{K}+$ et $\mathrm{Na}+$ sont données par les formules suivantes: al. 1995). Parmi les mécanismes de tolérance à la salinité figure la sélectivité vis-à-vis du potassium (Jeschke et al.1983). L'accumulation du potassium au détriment de sodium permet à la plante d'éviter les effets des déséquilibres nutritionnels induits par l'excès de sodium (Cramer et al.1985). En présence de $\mathrm{NaCl}$ dans le milieu, certains cations influent sur l'absorption du potassium et du sodium. Ainsi, une relation d'antagonisme a été établie entre $\mathrm{K}+$ et $\mathrm{Ca} 2+$ et entre $\mathrm{Mg} 2+$ et $\mathrm{Na}+$ (Soltani et al, 1990). Cette influence se caractérise par exemple par une accumulation du $\mathrm{Na}+$ dans les feuilles des plantes tolérantes comme la luzerne (Mezni et al.2002). Le travail présenté ici a pour objectif de décrire l'effet de la salinité sur la croissance et la distribution des principaux éléments minéraux chez le bananier.

$\mathrm{K}(\%)=$ lect $\times \mathrm{D} \times 0.92 / 100 \times \mathrm{m}$

$\mathrm{Na}+(\%)=$ lect $\times \mathrm{D} \times 0.92 / 100 \times \mathrm{m}$

Lect: lecture donnée par spectrophotométrie à flamme

D: Dilution

M: Masse de l'échantillon

Dosage de magnésium et du calcium (Benmiloud et Poloczanka, 1977)

Méthode et principe: Le dosage du Ca2+ et Mg2+ est effectué par spectrophotométrie d'absorption atomique à l'aide d'un (Spectrophotomètre d'absorption atomique Variant).

Dosage : Les teneurs en ces deux éléments sont données par les formules suivantes:

$\mathrm{Mg} 2+(\%)=\operatorname{axD} / \mathrm{mx} 0.01$

$\mathrm{Ca} 2+(\%)=\mathrm{axD} / \mathrm{mx} 0.01$

Dosage du phosphore ((Benmiloud et Poloczanka, 1977)

Méthode et Principe: Le dosage du $\mathrm{P}$ est réalisé par colorimétrie au moyen d'un Spectrophotomètre (modèle CECIL 1010) à une longueur d'onde de $825 \mathrm{~nm}$.

La teneur en phosphore est donnée par la formule suivante :

$P(\%)=A \times 0.058 \times 1 / \mathrm{m} \times 2.5$ 


\section{Belfakih et al... J. Appl. Biosci. 2013. Effet de la salinité sur la croissance des bananier et leur nutrition}

minérale au Maroc

\section{RÉSULTATS}

Effet du chlorure de sodium sur la production de biomasse : L'analyse de la variance relative à la production de matière sèche aérienne (MSA) (Tableau
1) révèle, aussi bien pour les variétés que pour les traitements salins, une différence hautement significative.

Tableau 1: Analyses de la variance relative à l'effet du $\mathrm{NaCl}$ sur la croissance des variétés de bananier.

\begin{tabular}{lll}
\hline Facteurs de variation & MSA & MSR \\
\hline Variétés (V) & $99,82^{* * *}$ & $104,15^{* * *}$ \\
Concentrations (C) & $159,98^{* * *}$ & $107,49^{* * *}$ \\
Vx C & $4,35^{*}$ & $7,88^{*}$ \\
\hline${ }^{* *}:$ Effet significatif au seuil de 1\% $;{ }^{* * *}:$ effet significatif au seuil de $0.1 \% ;$ & ${ }^{*}$ effet significatif au seuil de $5 \%$. \\
MSA = Matière sèche aérienne, MSR $=$ Matière sèche racinaire &
\end{tabular}

La réduction de biomasse aérienne est d'autant plus importante que la concentration en $\mathrm{NaCl}$ augmente, sauf à la concentration $2 \mathrm{~g} / \mathrm{l}$ on note une augmentation significative de la biomasse aérienne pour la variété grande naine, cette réduction débute dès la concentration $4 \mathrm{~g} / \mathrm{l}(-40 \%$ pour MSA) (figure1.a).

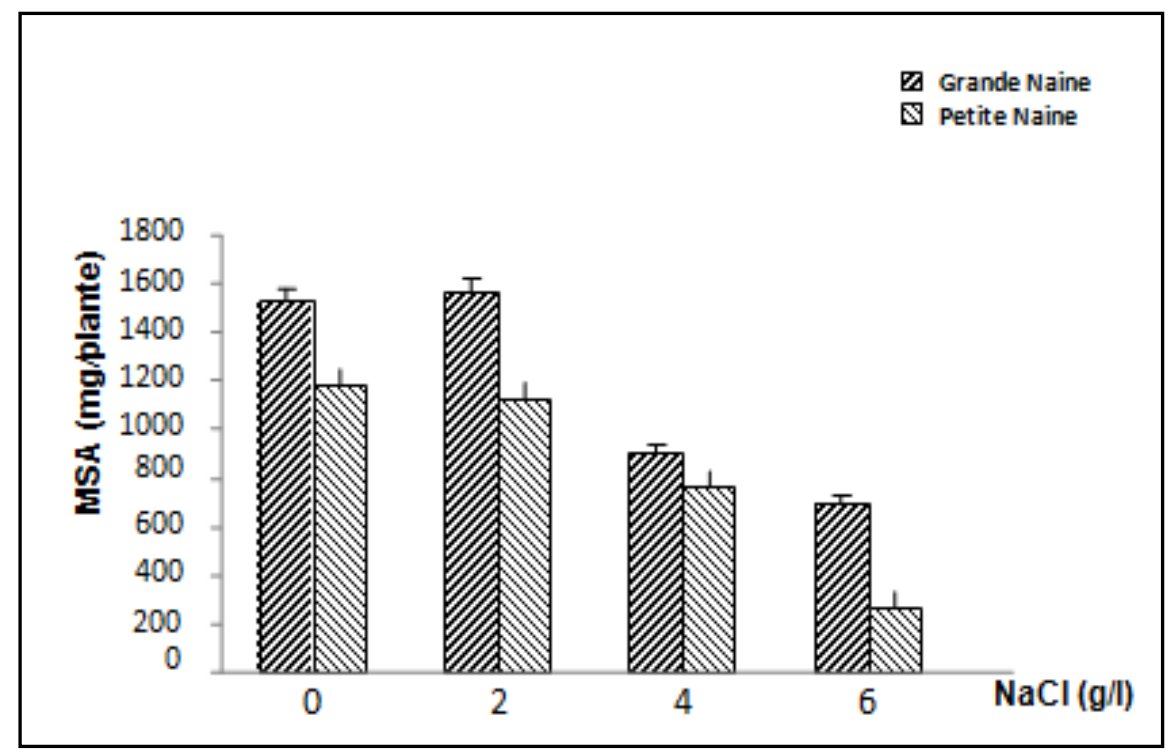

Figure 1.a : Effet du $\mathrm{NaCl}$ sur la production de matière sèche aérienne MSA

L'importance de cette diminution varie également en fonction des variétés étudiées. La variété grande naine se distingue de la variété petite naine par une MSA plus élevé, à la fois en absence et en présence de stress salin, une interaction variété $x$ salinité s'est révélée significative. L'effet du $\mathrm{NaCl}$ s'est traduit également par une réduction de la matière sèche racinaire (MSR) qui atteint $85 \%$ à $2 \mathrm{~g} / \mathrm{l}$ (Figure 1.b). Elle est surtout plus marquée à $6 \mathrm{~g} / \mathrm{l}$ avec une réduction de $70 \%$. La MSR, relativement plus affectée que la MSA est toutefois significativement réduite par la salinité ( $p$ $<0.001)$ et par la variété $(p>0,001)$ ( Tableau 1). La MSR de la variété petite naine est plus affectée que celle de la grande naine, une interaction variété $x$ salinité s'est révélée significative 


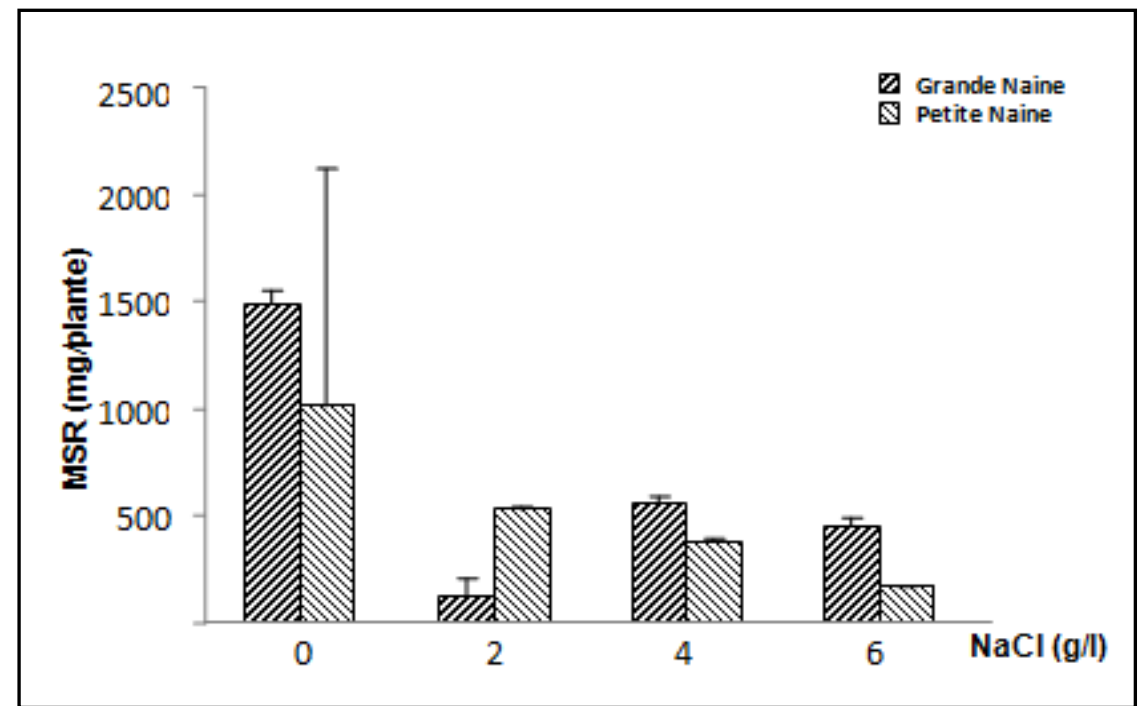

Figure 1.b : Effet du $\mathrm{NaCl}$ sur la production de matière sèche racinaire MSR

Effet de la salinité sur la composition minérale des différentes parties de la plante

Le sodium : L'analyse statistique de la teneur en sodium a révélé que l'effet de salinité est très hautement significatif (tableau 2) Les résultats relatifs à la teneur en sodium dans la partie aérienne montrent une augmentation progressive de la teneure en $\mathrm{Na}+$ avec l'augmentation de la concentration de $\mathrm{NaCl}$ du milieu. Les teneurs les plus élevées sont enregistrées chez la grande naine à la dose de $6 \mathrm{~g} / \mathrm{l} ;$ l'accumulation de cet élément est supérieure que la petite naine sauf à la dose $6 \mathrm{~g} / \mathrm{l}$ où l'accumulation de $\mathrm{Na}+$ devient plus importante par rapport à la petite naine (figure 2 .a).

Tableau 2 : Analyse de la variance relative à l'effet du $\mathrm{NaCl}$ sur la teneur en éléments minéraux $(\mathrm{N}, \mathrm{P}, \mathrm{K}, \mathrm{Mg}, \mathrm{Ca}$ et $\mathrm{Na}$ ) chez deux variétés de bananier.

\begin{tabular}{l|l|l|l|l|l|l}
\hline $\begin{array}{l}\text { Facteurs de } \\
\text { variation }\end{array}$ & $\mathbf{N}$ & $\mathbf{P}$ & $\mathbf{K +}$ & $\mathbf{M g 2 +}$ & $\mathrm{Ca} 2+$ & $\mathrm{Na}+$ \\
\hline Variétés $(\mathrm{V})$ & $0,01 \mathrm{NS}$ & $0,54 \mathrm{NS}$ & $23,52^{* * *}$ & $13,82^{* * *}$ & $6,27^{*}$ & $4,53^{*}$ \\
Concentration (c) & $2,57 \mathrm{NS}$ & $0,14 \mathrm{NS}$ & $26,52^{* * *}$ & $19,08^{* * *}$ & $21,52^{* * *}$ & $168,78^{* * *}$ \\
V. C & $4,92^{*}$ & $1,98 \mathrm{NS}$ & $11,91^{* * *}$ & $8,79^{* *}$ & $2,12 \mathrm{NS}$ & $1,52 \mathrm{NS}$ \\
\hline
\end{tabular}

${ }^{* *}$ : Effet significatif au seuil de $1 \% ;{ }^{* * *}$ : effet significatif au seuil de $0.1 \% ;{ }^{*}$ effet significatif au seuil de $5 \%$; NS : effet nom significatif ; Les chiffres correspondent aux valeurs du F calculé 


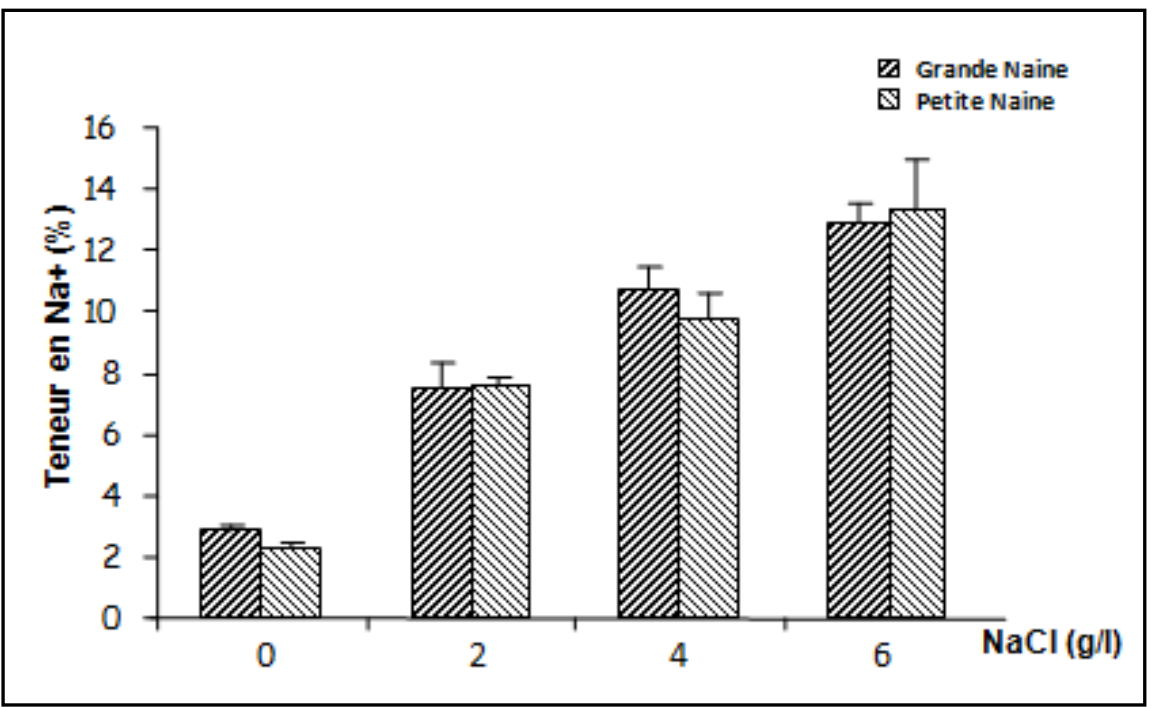

Figure 2.a : Effet du $\mathrm{NaCl}$ sur la teneur en sodium dans les parties aériennes

La comparaison des teneurs en $\mathrm{Na}+$ des parties aérienne et racinaire montre que l'augmentation progressive de $\mathrm{Na}+$ dans la partie aérienne reste inférieure à celle observée dans la partie racinaire; cependant des teneurs égales en $\mathrm{Na}+$ sont enregistrées dans la partie aérienne.

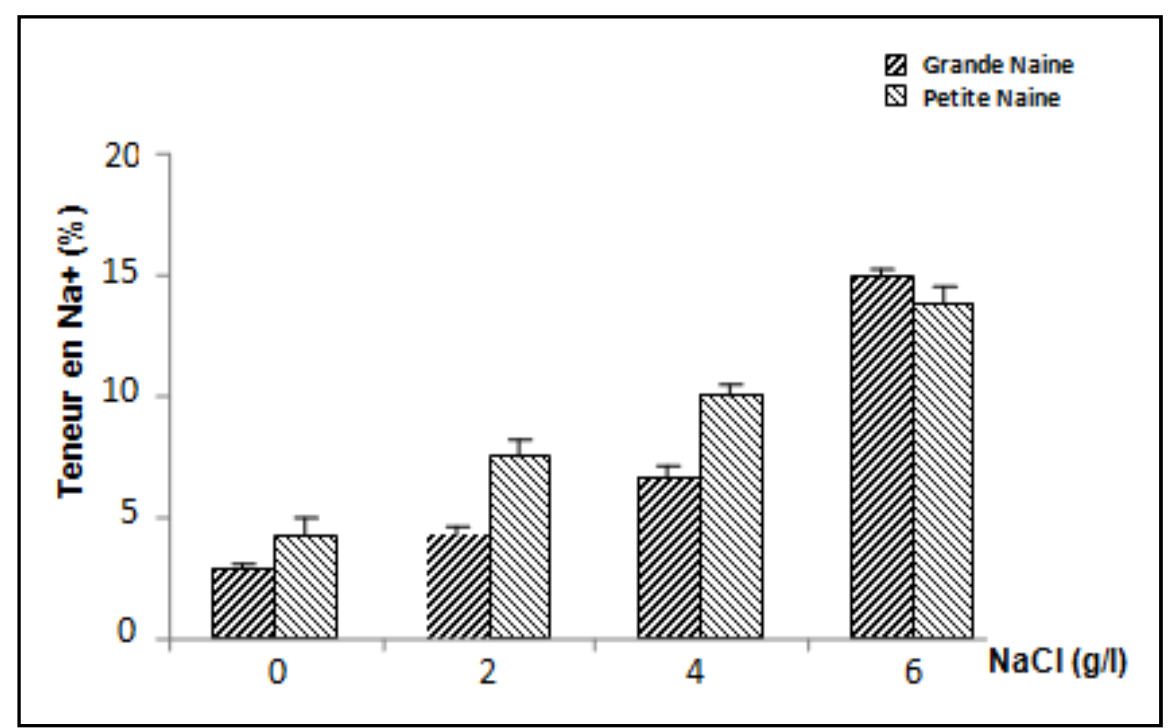

Figure 2.b: Effet du $\mathrm{NaCl}$ sur la teneur en sodium dans les parties racinaires

Le Potassium : Pour la partie aérienne L'examen de la figure 3 montre que la teneur en $\mathrm{K}+$ augmente significativement avec le traitement salin, Elle est plus élevée chez la petite naine que chez la grande naine à $6 \mathrm{~g} / \mathrm{l}$. En effet c'est la grande naine qui montre les valeurs les plus élevées à la dose de $4 \mathrm{~g} / \mathrm{l}$ elle est $16 \%$ pour la partie aérienne et $14 \%$ pour la partie racinaire.
Les teneurs les plus faibles en $\mathrm{K}+$ sont observées chez la petite naine en milieu témoin elle de $7 \%$ pour la partie aérienne et $8 \%$ pour la partie racinaire, tandis que les deux variétés ont presque la même teneur en $\mathrm{K}+$ au milieu à $2 \mathrm{~g} / \mathrm{l}$ de $\mathrm{NaCl}$. 


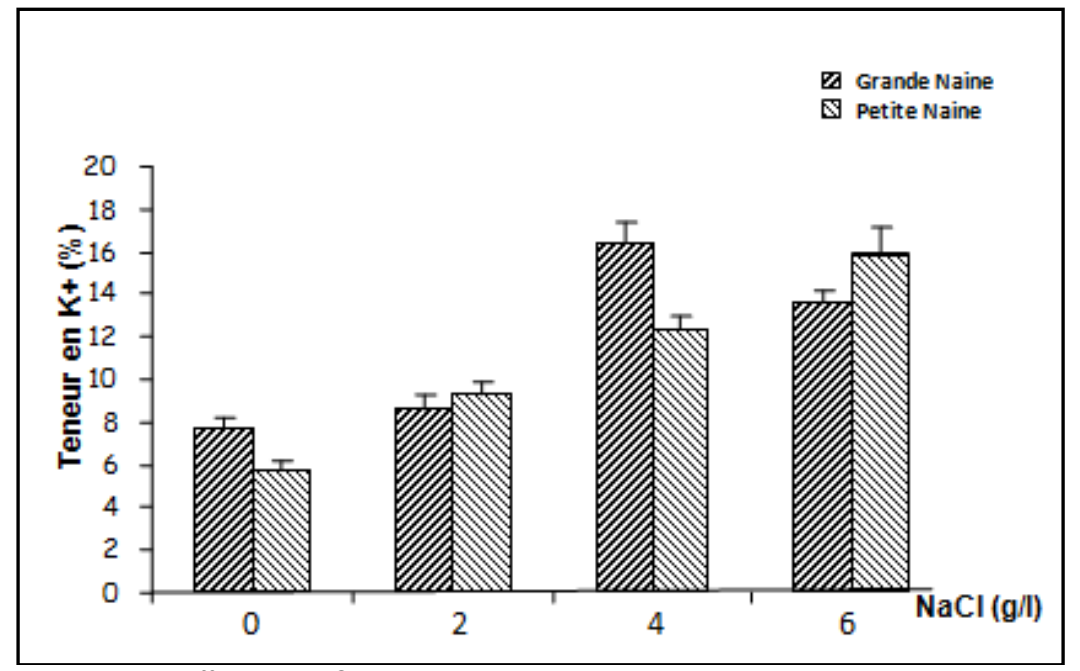

Figure 3.a: Effet du $\mathrm{NaCl}$ sur la teneur en Potassium dans les parties aériennes

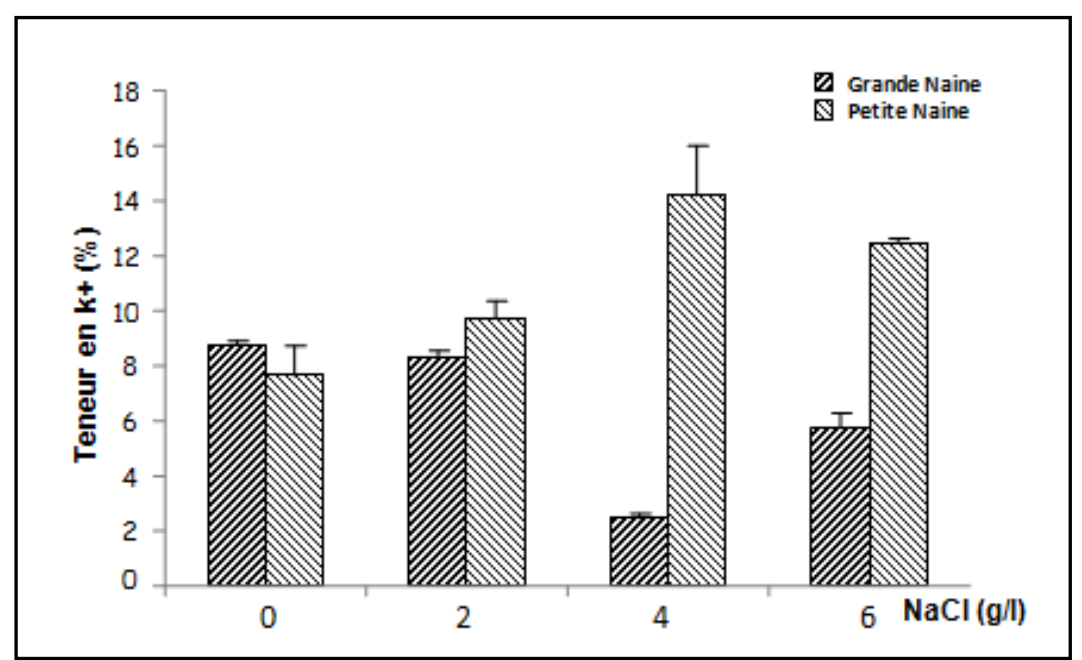

Figure 3.b: Effet du $\mathrm{NaCl}$ sur la teneur en Potassium dans les parties racinaires

Ainsi la comparaison des traitements salins chez les deux variétés montre une différence très hautement significative vis-à-vis l'accumulation de ce cation (Tableau 2). Pour les racines et contrairement à la partie aérienne, la salinité réduit significativement la teneur en $\mathrm{K}+$ chez la grande naine, même s'il y a une augmentation remarquable à $6 \mathrm{~g} / \mathrm{l}$, Tandis que chez la petite naine la teneur en cet élément augmente très significativement avec le l'augmentation de la salinité (Tableau 2). La teneur la plus faible en $\mathrm{K}+$ est enregistré à $4 \mathrm{~g} / \mathrm{l}$ chez la grande naine et la plus forte chez la petite naine. Généralement il y a une différence très hautement significative entre les deux variétés et les concentrations vis-à-vis l'absorption de K+ (Tableau 2).

Le calcium :A travers les résultats illustrés dans la figure 4, l'augmentation de la concentration saline dans le milieu est accompagnée d'une diminution très hautement significative chez les deux variétés en $\mathrm{Ca} 2+$ (Tableau 2) dans la partie aérienne. Toutefois à $4 \mathrm{gl} / \mathrm{l}$ de $\mathrm{NaCl}$, on assiste à une accumulation très remarquable de $\mathrm{Ca}++$ dans la partie racinaire, la variété petite naine se distingue de la variété grande naine par le maintien d'une teneur importante et significative en $\mathrm{Ca} 2+$ en particulier dans les racines. 


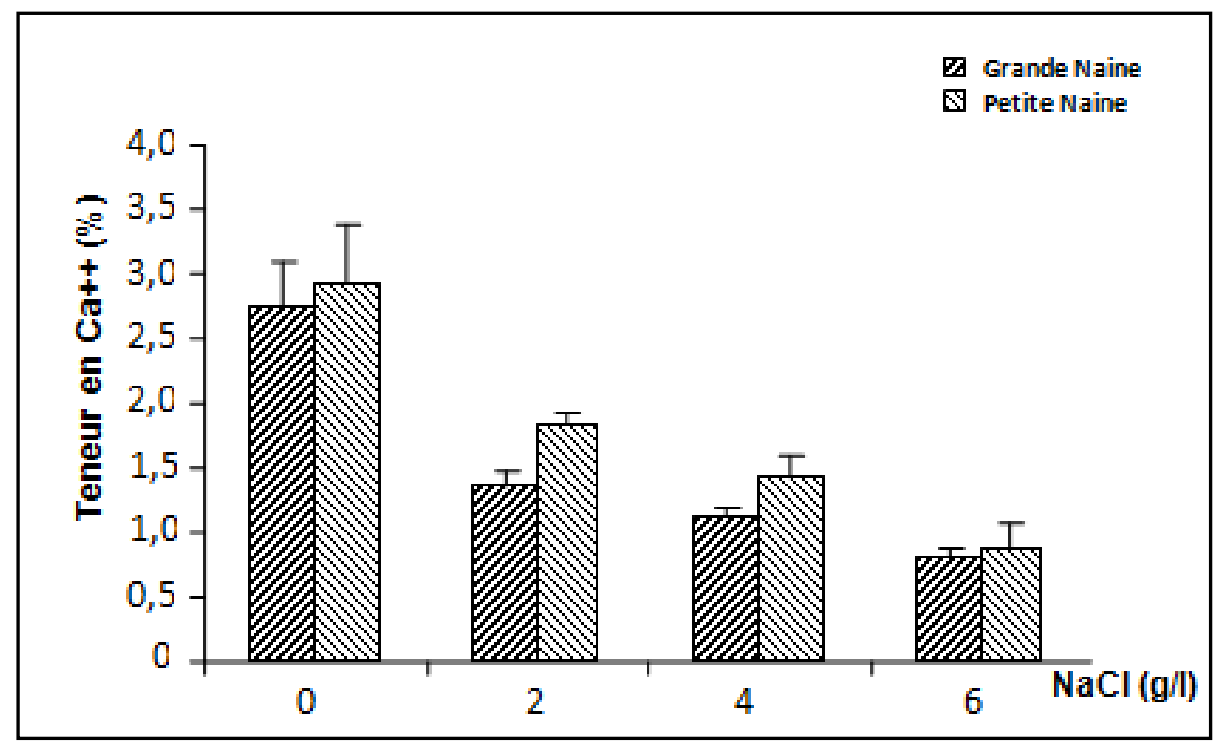

Figure 4 .a: Effet du $\mathrm{NaCl}$ sur la teneur en Calcium dans les parties aériennes

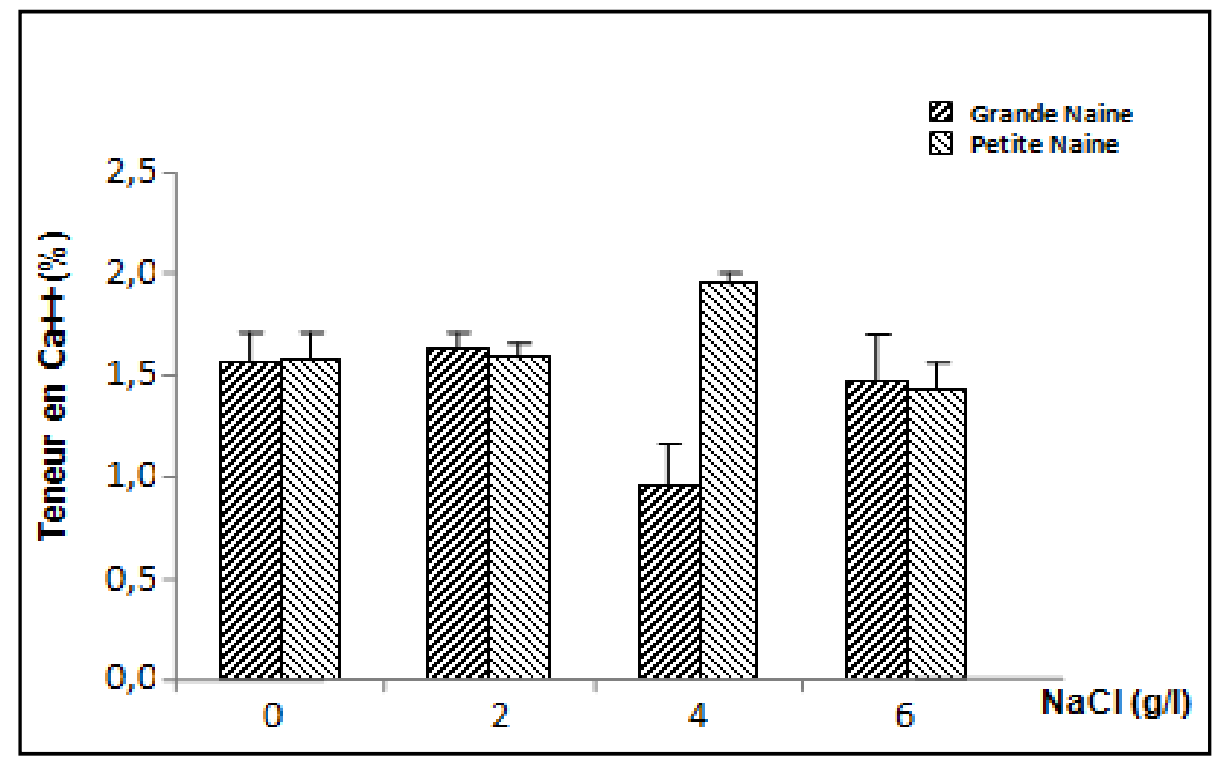

Figure 4.b : Effet du $\mathrm{NaCl}$ sur la teneur en Calcium dans les parties racinaires

Le magnésium : L'examen de la figure 5 montre que la teneur en $\mathrm{Mg} 2+$ dans la partie aérienne devient significativement faible chez les deux variétés (Tableau 2), A la concentration $2 \mathrm{~g} / \mathrm{l}$, on enregistre par contre une accumulation de Mg2+ chez la petite naine. Au niveau racinaire Les teneurs en $\mathrm{Mg} 2+$ diffèrent significativement selon les variétés et selon les concentrations (Tableau 2). pour la grande naine : I' accumulation de $\mathrm{Mg} 2+$ diminue régulièrement avec la concentration saline du milieu tandis que chez la petite naine on note une augmentation à $2 \mathrm{~g} / \mathrm{l}$ : alors que a $4 \mathrm{~g} / \mathrm{l}$ et $6 \mathrm{~g} / \mathrm{l}$ la teneur de cet ions devient moins importante. 
Belfakih et al... J. Appl. Biosci. 2013. Effet de la salinité sur la croissance des bananier et leur nutrition minérale au Maroc

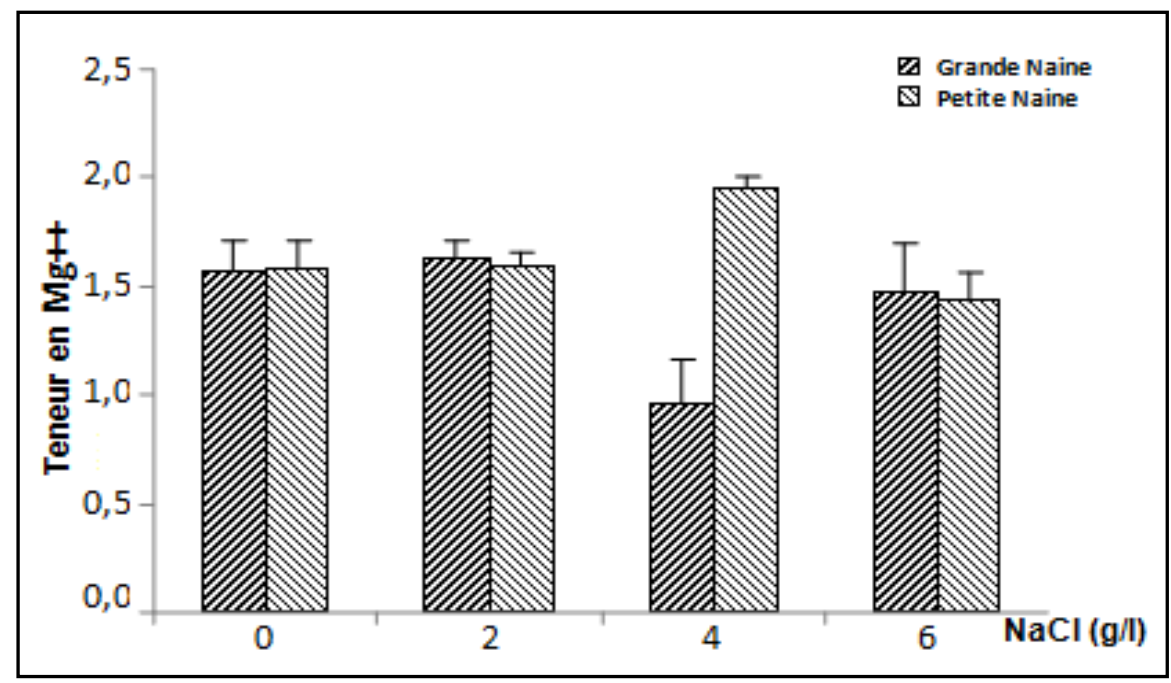

Figure 5.a: Effet du $\mathrm{NaCl}$ sur la teneur en Magnésium dans les parties aériennes

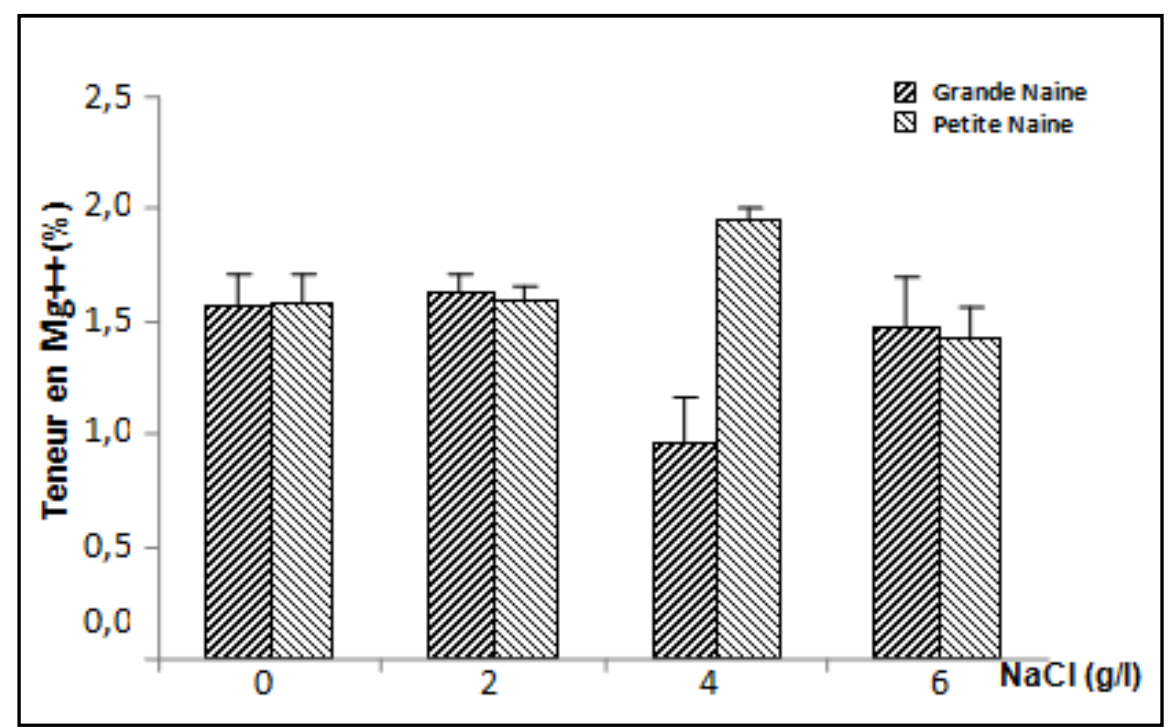

Figure 5.b: Effet du $\mathrm{NaCl}$ sur la teneur en Magnésium dans les parties racinaires

La teneur en azote et en phosphore varie peu avec l'augmentation de la concentration de $\mathrm{NaCl}$ chez les deux variétés, aucune différence significative n'est enregistrée pour les variétés et pour les concentrations salines (Tableau 2 ; Figures 6 et 7). 
Belfakih et al... J. Appl. Biosci. 2013. Effet de la salinité sur la croissance des bananier et leur nutrition minérale au Maroc

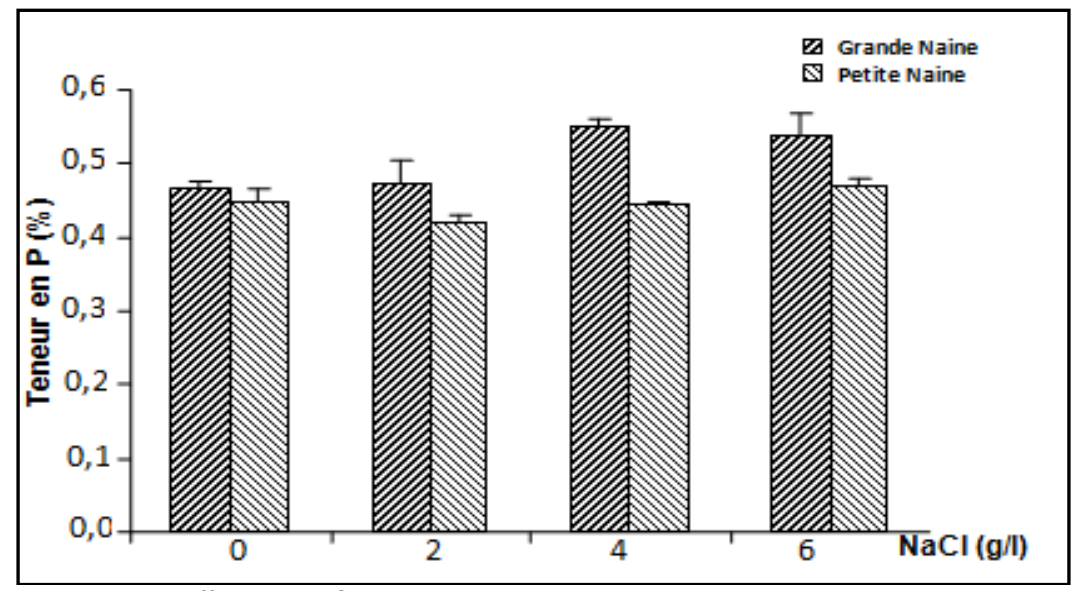

Figure 6.a: Effet du $\mathrm{NaCl}$ sur la teneur en Phosphore dans les parties aériennes

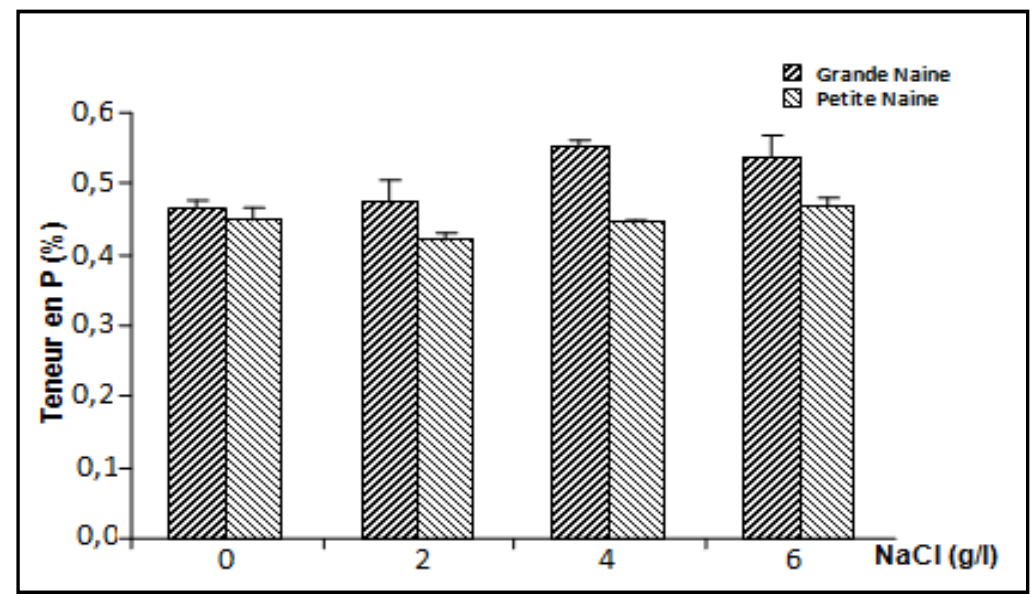

Figure 6.b: Effet du $\mathrm{NaCl}$ sur la teneur en Phosphore dans les parties racinaires

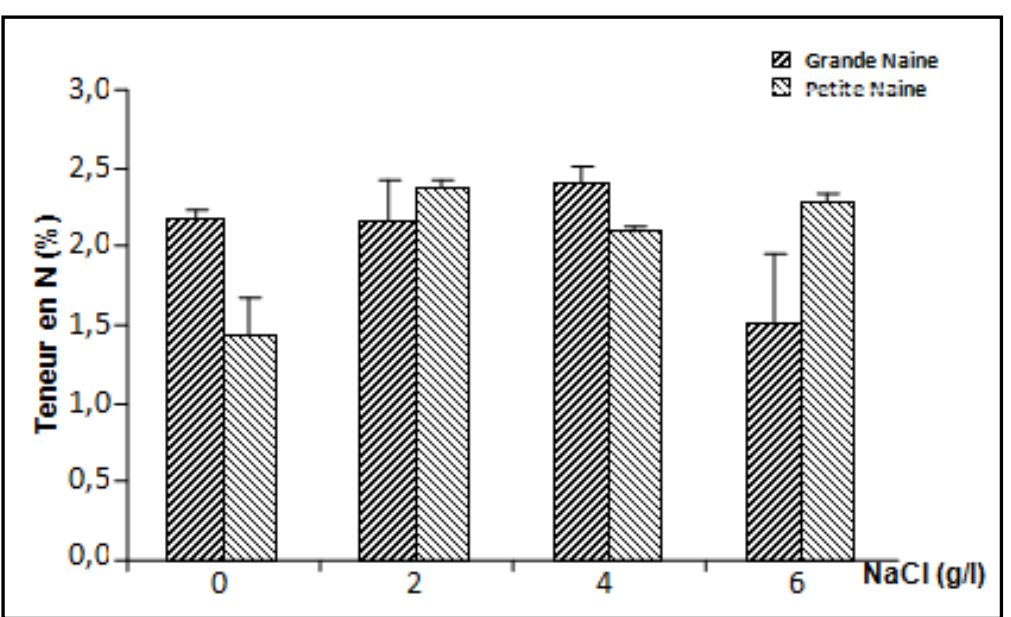

Figure 7: Effet du $\mathrm{NaCl}$ sur la teneur en Azote dans les parties aériennes 
DISCUSSION

Différence génotypique de sensibilité à la salinité : La réduction des biomasses aériennes et racinaires, observée chez le bananier sous l'effet des fortes Concentrations en $\mathrm{NaCl}$ (photo1) a été rapportée par plusieurs auteurs chez différentes espèces dont le blé (
Isla et al. , 1998), l'orge (EL Mekkaoui et al .,1994), le sorgho (Igartua et al. ,1995), le tournesoll (El Midaoui et al ., 1999) la luzerne (Mezni et al. ,2002; Ibriz et al. , 2005), et la vigne (Alexander et al. , 2003 ; Walker et al. , 2003 ; Charbaji et al. , 2004).

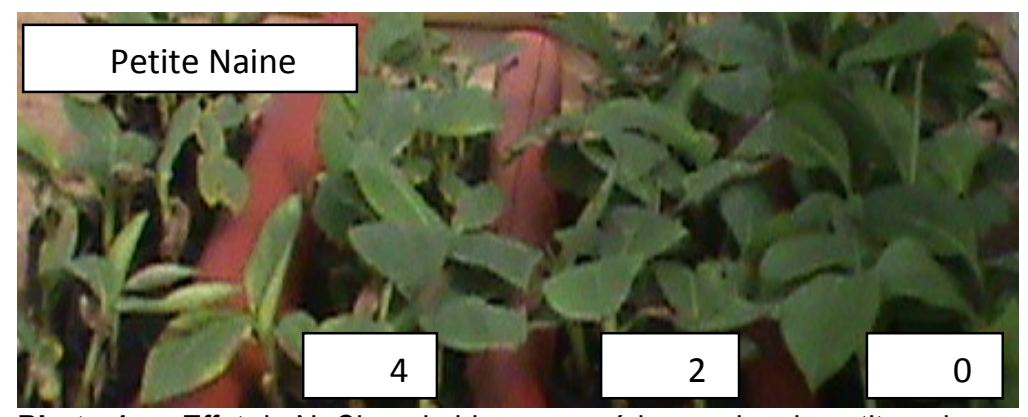

Photo 1a : Effet du $\mathrm{NaCl}$ sur la biomasse aérienne chez la petite naine

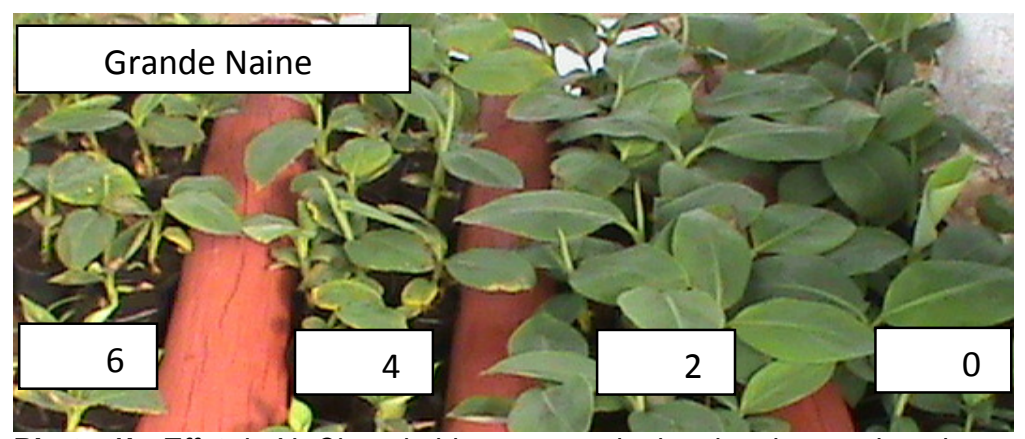

Photo 1b: Effet du $\mathrm{NaCl}$ sur la biomasse racinaire chez la grande naine

Cette réduction peut être liée à des perturbations de concentrations des régulateurs de croissance (acide abscissique et cytokinines comme décrit par Termaat et al. 1985 et Kuiper et al. 1990., mais aussi à une réduction de la capacité photosynthétique suite à une diminution de la conductance stomatique du $\mathrm{CO} 2$ induite par la contrainte saline (Walker et al.1981; Santiago et al. ; 2000). Chez Citrus aurantium, cette réduction de croissance serait principalement due à l'effet osmotique de la salinité (Ruiz et al. 2001). Ainsi, on peut exclure que l'effet inhibiteur de $\mathrm{NaCl}$ sur la croissance passe par une perturbation de l'alimentation en eau. La variété grande naine se distingue de la variété petite naine par une MSA plus élevée. Ce résultat concorde avec des résultats trouvés sur une variété du bananier 'Pacovan' du Brésil qui a maintenu une production de biomasse élevée à niveau des salinités importants, elle a maintenu une forte production de biomasse au niveau de salinité le plus élevé $(100 \mathrm{~mol} / \mathrm{m} 3)$ (Waked Ferreira Gomes, 2002).Ceci laisse penser que la variété grande naine est peu tolérante à la salinité que la petite naine. Ainsi, la plante semble s'adapter au stress salin en réduisant en premier lieu son système racinaire préservant la partie aérienne afin de maintenir et assurer le fonctionnement photosynthétique. Les résultats de notre étude montrent que les réductions des biomasses aériennes et racinaires seraient probablement dues à une différence d'accumulation des ions minéraux entre les différentes parties de la plante.

Les teneurs en éléments minéraux : La salinité a réduit les teneurs en $\mathrm{Ca} 2+$ et $\mathrm{Mg} 2+$ et a accentué l'accumulation de $\mathrm{Na}+$ et $\mathrm{K}+$. L'accumulation de $\mathrm{Na}+$ est moindre dans la partie aérienne que la partie racinaire chez la grande naine, des résultats similaires ont été rapportés par plusieurs auteurs (Waked Ferreira 


\section{Belfakih et al... J. Appl. Biosci. 2013. Effet de la salinité sur la croissance des bananier et leur nutrition minérale au Maroc}

Gomes, 2002). Pour des génotypes de bananier; à priori la protection des parties aériennes contre l'envahissement par le $\mathrm{Na}+$ peut être assurée par un contrôle de l'absorption par les racines. Des plantes sensibles qui sont moins efficaces dans l'absorption du $\mathrm{Na}+$ et son transport vers le xylème mais qui sont par contre plus efficaces dans la rétention du $\mathrm{Na}+$ dans leurs tissus (Salma, 1986). Ceci explique dans certaine mesure le fait que la teneur en $\mathrm{Na}+$ soit élevée dans les racines. L'accumulation de $\mathrm{Na}+$ au niveau des racines semble s'expliquer d'une part par une aptitude d'utiliser l'ion pour l'ajustement osmotique, d'autre part pour qu'il soit probablement exclu au niveau des racines. Un comportement de type "exclusive " est donc affiché et adopté par le bananier. Nos résultats indiquent que l'exclusion de sels s'effectue au niveau des racines. Le même résultat a été rapporté par ( Waked Ferraira Gomes, 2002) chez trois variétés du bananier «Pacovan), FHIA et Caipera et par Ouakardous (1999) chez la grande naine au groupe Cavendish. La diminution de la teneur en $\mathrm{K}+$ des parties racinaires chez la grande naine est associée à une augmentation de cette teneur dans les parties aériennes. Ceci semble indiquer un comportement d'ajustement osmotique (surtout après l'invasion des ions sodium) qui, par conséquent, assure la survie des plants, La plante adsorbe et véhicule le maximum de potassium vers la partie aérienne afin d'assurer un ajustement osmotique. II semble que c'est surtout au niveau des feuilles que la plante rétablit l'équilibre de son potentiel osmotique via l'ion potassium et atténue ainsi le stress osmotique engendré par $\mathrm{NaCl}$. La capacité de la plante à pomper le potassium de la solution nutritive, puis de le véhiculer vers les feuilles, représente une stratégie d'adaptation au stress salin via la régulation osmotique par l'ion potassium. Ces résultats pourraient indiquer une sélectivité vis-à-vis de

\section{CONCLUSION}

Les caractéristiques ioniques de la variété de bananier, la grande naine, diffèrent de celles de la petite naine, La salinité a réduit les teneurs en $\mathrm{Ca} 2+$ et $\mathrm{Mg} 2+$ et a accentué l'accumulation de $\mathrm{Na}+$ et de $\mathrm{K}+$. L'accumulation de $\mathrm{Na}+$ est moindre dans la partie aérienne que dans la partie racinaire chez la grande naine, qui montre une sélectivité très importante vis-àvis du potassium, elle est ainsi considérée comme une espèce tolérante à la salinité. Les accumulations de K+
$\mathrm{K}+$ aux fortes concentrations en $\mathrm{NaCl}$. Cette sélectivité est plus marquée aussi chez la petite naine. Des résultats similaires ont été rapportés par $E$. Waked Ferreira Gomes, et al. , (2002) chez la banane, et par Lamia Hamrouni et al. (2010) chez la vigne. L'importance de l'augmentation de la teneur en $\mathrm{K}+$ pourrait être donc un indicateur de la tolérance au sel. La sélectivité en faveur de $\mathrm{K}+$ a été rapporté par plusieurs auteurs (Bizid et al. 1988; El Mekkaoui, 1992). La réduction de la teneur en Ca2+ en présence de $\mathrm{NaCl}$ varie significativement entre les deux variétés. La teneur en Ca2+ aérienne diminue plus rapidement avec l'augmentation de la concentration de $\mathrm{NaCl}$. Chez la petite naine que la grande naine. Les mêmes résultats sont rapportés par ( Ferraira Gomes, 2002), chez trois variétés du bananier Pacovan, FHIA et Caipera. Ceci suggère que le niveau de tolérance d'une espèce pourrait être également lié à sa teneur racinaire en Ca2+ (Pourrat et Dutuit, 1994). Bien qu'une baisse des concentrations de magnésium ait été enregistrée au niveau des feuilles et des racines, il ne semble pas que cet élément soit un critère capital quant à l'adaptation des plantes au stress salin. Les quantités accumulées ne reflètent pas non plus un aspect particulier de la tolérance à la salinité. Le rapport K/Na affiche une tendance générale à la baisse, dans les parties aériennes et racinaires pour les deux variétés. $C e$ résultat pourrait indiquer une perte de sélectivité vis-à-vis du $\mathrm{K}+$ aux fortes concentrations en $\mathrm{NaCl}$, cette réduction est plus remarquable dans les parties racinaires chez la variété grande naine qui peut expliquer l'augmentation du k+ dans la partie aérienne de cette variété. Ainsi, le potassium est très sollicité au niveau du compartiment aérien pour la réalisation des métabolismes cellulaires où il est généralement employé comme cofacteur dans les réactions enzymatiques et biochimiques.

et $\mathrm{Na}+$ ainsi que la réduction de certains éléments comme le Ca2+et le Mg2+ peuvent s'expliquer, en partie, par la réduction de la matière sèche des parties aériennes et racinaires. La tolérance observée chez les variétés du bananier pourrait être liée à une bonne sélectivité vis-à-vis du potassium. Ce travail met en exergue la complexité de l'étude de la nutrition minérale, sachant que cette dernière aide à une compréhension partielle de la tolérance à la salinité. 


\section{Belfakih et al... J. Appl. Biosci. 2013. Effet de la salinité sur la croissance des bananier et leur nutrition minérale au Maroc}

Les résultats obtenus confirment que l'amélioration de la productivité des bananiers au Maroc ne peut être réalisée que par des travaux destinés à élaborer un matériel génétique adapté à partir de la variété grande RÉFÉRENCES BIBLIOGRAPHIQUES

Anonyme(2005), "Situation du secteur de la Banane au Maroc". Pack info. №36 25-29.

Benmiloud M, Poloczanka WA, 1977. "Pratique du diagnostique foliaire : laboratoire générale de chimie". MARA Direction de la Recherche Agronomique (Maroc).

Bizid $\mathrm{E}$, Grigon $\mathrm{C}, 1988$. Tolérance à $\mathrm{NaCl}$ et sélectivité $\mathrm{K}+/ \mathrm{Na}+$ chez les triticales ". Agronomie 8 (1) 23-27.

Bohra JS, Doerffling K, 1993, "Potassium nutrition of rice (Oryza sativa L.) varieties under $\mathrm{NaCl}$ salinity". Plant and Soil 152 299-303.

CA/MADR, 2003. "Journée de sensibilisation sur les alternatives au bromure de méthyle dans la désinfection du sol du bananier. Chambre d'Agriculture, Ministère de l'Agriculture et du Développement Rural". 20 p.

Charbaji T, Ayyoubi Z, 2004. "Differential growth of some grapevine varieties in Syria in response to salt in vitro". In Vitro Cell. Dev. Biol. Plant, 40 221-224.

Cramer GR, Lauchi A, Polito EVS, 1985. "Deplacement of $\mathrm{Ca}+$ by $\mathrm{Na}+$ from the plasmalemme of root celle". A primary reponse to Salt stress. Plant physiol. 83 510-516.

DPV/DH/MARA, 1993. "Rapport sur le bananier. Bilan de la campagne agricole 1992/1993". Direction de la production végétale. Division de I'horticulture. Ministère de l'Agriculture et de la Réforme graire, Maroc, $15 \mathrm{p}$.

El Mekkaoui M, 1992. "Étude des caractères physiologiques d'adaptation à la salinité chez trois céréales: le blé dur ( $T$. durum Desf.), l'orge (H. vulgar L.) et le triticale (T. durum $x$ secale céréale)". Thèse de Doctorat èsSciences Agronomiques, I.A.V. Hassan II, Rabat.

EL Mekkaoui M, Agbani M, Monneveux P, 1994."Rôle de la sélectivité $\mathrm{K}+/ \mathrm{Na}+$ et de l'accumulation de proline dans l'adaptation à la salinité chez l'orge ( Hordeum vulgar L.) et du blé dur ( Triticum durum Desf.)". Acte Inst. Agron. Vét. (Maroc), 14(2) 27-36. naine. Le choix doit s'effectuer sur la base d'un grand nombre de caractères (morphologiques, biochimiques physiologiques) contrôlant les déterminismes génétiques complexes de la tolérance à la salinité.

El Midaoui M, Talouizte A, Benbella M, Serieys $H$, Berville A, 1999. "Responses of live sunflower genotypes (Helianthus annus L.) to different concentrations of sodium chloride". Helia. 22(30) 125-138.

Ftouhi A, 1981. "Les sols salés dans les offices régionaux de mise en valeurs agricoles". Mémoire de fin d'études. E.N.A. Meknèse.

Galàn SV, Ait Oubahou A, Abdelhaq H, 2004. "Culture de bananes sous Serres". Musalit, 123 86-95.

Richards RA, 1992. "Increasing salinity tolerance of grain crops: is it worthwhile?" Plant and Soil 146 89-98.

Greenway E, Munns R, 1980. "Mechanisms of salt tolerance in nonhalophytes". Ann. Rev Plant physiol. 30 (1980) 149-190.

Hamrouni L, Hanana M, Abdelly $C$ e Ghorbel A, 2011Exclusion du chlorure et inclusion du sodium : deux mécanismes oncomitants de tolérance à la salinité chez la vigne sauvage Vitis vinifera subsp. sylvestris (var.'Séjnène)", Biotechnol. Agron. Soc. Environ. 15(3) 387400.

Ibriz M, Thami Alami I, Zenasni L, Alfaiz C et Benbella $\mathrm{M}, 2005$. "Effet de la salinité sur le rendement en biomasse et la composition en éléments minéraux d'écotypes marocains de luzerne (Medicago sativa L.) » Al awamia Vol. $2 \mathrm{~N}^{\circ} 3$.

Igartua E, Garcia MP Lasa JM, 1955. "Field responses of grain sorghum to salinity gradient". Field Crop Research 42 (1) 15-25.

Jeschke WD, 1983. "Cations fluxes in relation to specific and varietal differences". Plant and Soil, 72 197-212.

Kuiper D, Schuit D, Kuiper JC, 1990. "Actual cytokinin Concentrations in plant tissue as an indicator for salt resistance in cereal". Plant Soil, 123 243-245.

Mezni M, Albouchi A, Bizid E, Hamza M, 2002. "Effet de la salinité des eaux d'irrigation sur la nutrition minérale chez trois de luzerne pérenne ( Medicago sativa L.)". Agronomie 22 283-291. 


\section{Belfakih et al... J. Appl. Biosci. 2013. Effet de la salinité sur la croissance des bananier et leur nutrition minérale au Maroc}

Morchid A, 1999. Potentialités de développement des cultures sous abri-serres dans la zone du périmètre du Gharb". Mém. 3ème cycle. Institut Agronomique et Vétérinaire Hassan II, Rabat, Maroc 161 p. Norse D. 1974. Plant diseases in Barbados. Phytopathol. Pap. 181 38.

Ouakardous M, 1999. "Effet de $\mathrm{NaCl}$ sur la croissance du bananier (CV, grande et petite naine) et dur le contrôle de niveau de population de deux espèces de nématodes (Meloidogcine spp et radopholus similis)". Mémoire en troisième cycle en agronomie, I. A. V. Complex Horticole d'Agadir.

Pinta M, 1971. "Spectrométrie d'absorption atomique". Applications à l'analyse chimique. Tome II.

Pourrat $Y$, Dutuit $P, 1994$. "Étude précose des effets morphologiques et physiologiques du rapport sodium/calcium in vitro sur une population d'Atriplix halimus. Quel avenir pour l'amélioration des plantes ?" Ed. AUPELFUREF. John Libbey Eurotext,Paris, 283-295.

Ruiz-Medrano R, Xoconostle C Lucas WJ, 2001. "The phloem as a conduit for inter-organ communication. Curr. Opin. Plant Biol., 4 202209.

Santiago LS, Lau TS, Melcher PJ, Steele OC, Goldstein G, 2000. "Morphological and physiological responses of Hawaiian Hibiscus tiliaceus populations to light and salinity". Int. J. Plant Sci., 161 99-106.

Slama F, 1986. "Intervention des racines dans la sensibilité ou la tolérance à $\mathrm{NaCl}$ des plantes cultivées". Agronomie, 7 (6) 651-658.

Soltani A, Hajji M, Grignon C, 1990. "Recherche de facteurs limitant la nutrition minérale de l'orge en milieu salé. Agronomie 10 857-866.

SRPV/MARA, 1981. "Rapport sur le bananier. Service régional de la Protection des végétaux". Ministère de l'Agriculture et de la Réforme Agraire, $10 \mathrm{p}$.

Storey R, 1995. "Salt tolerance, ions relations and the effect of root medium on the response of Citrus to salinity”. Aust. J. Plant Pysiol. 22 101114.

Szablocs I, 1994. "Soils ans salinization". In: Hand book of plant and Crop stress. Ed. Mohammed Pessarakli 3-7.
Termaat A, Passioura JB, Munns R, 1985. "Shoot turgor does not limit shoot growth of $\mathrm{NaCl}$ affected wheat and barley". Plant Physiol., 77 869-872.

Waked Ferreira Gomes E, Willadino L, Semen Martins LS, Rangel Camara T, Oliveira Silva S, 2002. "Génotypes de bananiers (Musa spp.) soumis au Stress salin: tolérance et sensibilité". INFOMUSA- 213 (11).

Walker RR, Torokfalvy E, Scott NS, Kriedemann PE, 1981. "An analysis of photosynthetic response to salt treatment in Vitis vinifera". Aust. J. Plant Physiol., 8 (359-374.

Walker RR, 2003 "Salinity effects on vines and wines". Bull. OIV, 76 201-227. 